\title{
Clinico-pathological profile of acute promyelocytic leukaemia at Al-Amal oncology-haematology centre, Qatar
}

F.A. Ibrahim, ${ }^{1}$ M.A.Yassin, ${ }^{2}$ H.R. El-Ayoubi, ${ }^{2}$ I.A. Alhijji, ${ }^{2}$ A.S. Albinali, ${ }^{1}$ S.M. Almansourl and F.M. Qafoud ${ }^{1}$

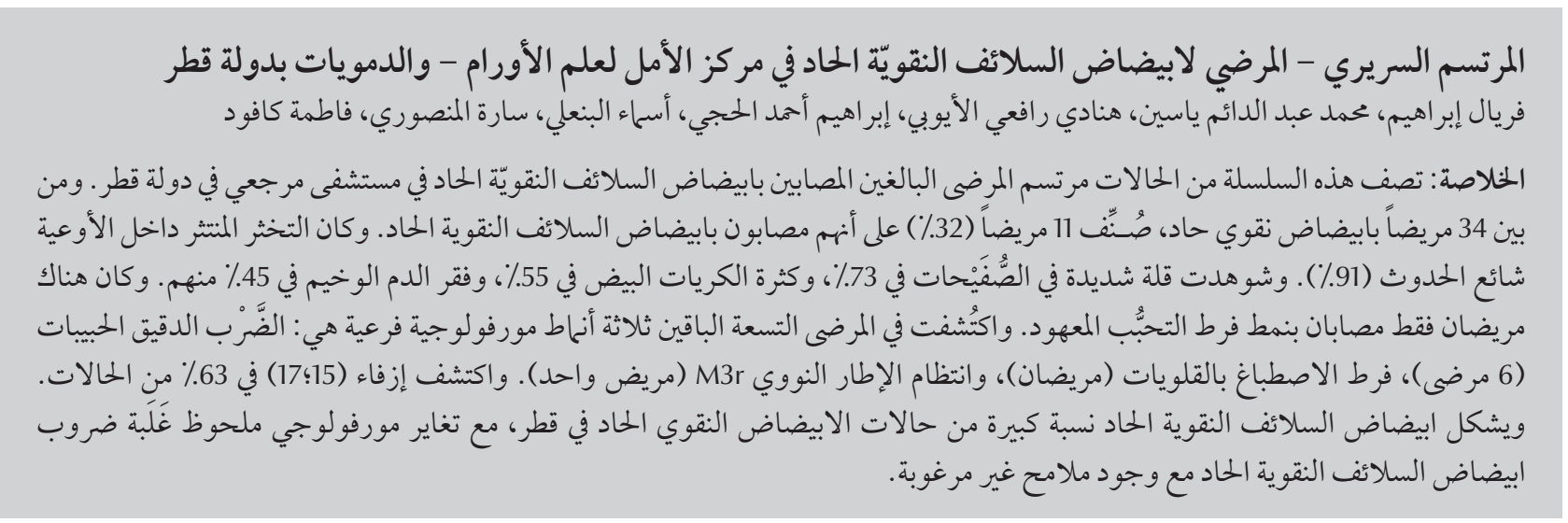

ABSTRACT This cases series describes the profile of adult patients with acute promyelocytic leukaemia (APL) at a referral hospital in Qatar. Of 34 acute myeloid leukaemia (AML) cases diagnosed, 11 (32\%) were classified as APL. Disseminated intravascular coagulation was common at presentation (91\%). Severe thrombocytopenia was seen in $73 \%$, leukocytosis in 55\% and severe anaemia in 45\%. Only 2 patients were of the classic hypergranular type. In the remaining 9 patients, 3 morphological subtypes were recognized: microgranular variant (6 patients), hyperbasophilic (2 patients) and regular nuclear outline M3r (1 patient). Translocation t $15 ; 17)$ was detected in $63 \%$ of cases. APL constitutes a high proportion of AML cases in Qatar, with considerable morphological heterogeneity and a predominance of APL variants with unfavourable presenting features.

Profil anatomoclinique de la leucémie promyélocytaire aiguë établi au centre d'oncologie et d'hématologie d'Al-Amal (Qatar)

RÉSUMÉ Cette série de cas décrit le profil des patients adultes atteints de leucémie promyélocytaire aiguë et traités dans un hôpital de recours au Qatar. Sur 34 cas diagnostiqués de leucémie myéloïde aiguë, 11 d'entre eux (soit $32 \%)$ ont été classés comme leucémie promyélocytaire aiguë. Une coagulation intravasculaire disséminée a été fréquemment constatée au moment de la présentation du patient (91\%). Une thrombopénie sévère a été observée dans $73 \%$ des cas, une leucocytose dans $55 \%$ des cas et une anémie sévère dans $45 \%$ des cas. Seuls deux patients présentaient une forme classique hypergranulaire. Chez les neuf autres patients, trois sous-types morphologiques ont été identifiés : une variante microgranulaire (six patients), une variante hyperbasophile (deux patients) et une variante $\mathrm{M} 3$ avec contour nucléaire régulier (un patient). Une translocation t $(15 ; 17)$ a été détectée dans 63 \% des cas. La leucémie promyélocytaire aiguë constitue une proportion importante des cas de leucémie myéloïde aiguë au Qatar, et se caractérise par une hétérogénéité morphologique importante et une prédominance des variantes de leucémie promyélocytaire aiguë avec signes d'appel défavorables.

${ }^{7}$ Department of Laboratory Medicine and Pathology; ${ }^{2}$ Department of Haematology and Bone Marrow Transplant, Al-Amal Hospital, Qatar (Correspondence to M.A. Yassin: yassin160@yahoo.co.uk).

Received: 13/01/09; accepted: 12/03/09 


\section{Introduction}

Acute promyelocytic leukaemia (APL) is a subtype of acute myeloid leukaemia (AML) that has unique morphological, cytogenetic and molecular features [1]. These include a potentially devastating coagulopathy, which carries a high risk of mortality [2], and sensitivity to retinoid-differentiating agents including all-trans-retinoic acid (ATRA) [3]. The use of ATRA, together with conventional chemotherapy, has substantially increased the number of patients who can be cured of APL. The impressive improvement in treatment outcomes observed with ATRA is, at least in part, due to the rapid correction of the coagulopathy, implying that the administration of the drug must occur at the beginning of treatment [4]. Thus, rapid diagnosis of APL is critical for treatment decisions since ATRA must be given promptly.

Unequivocal diagnosis of APL can only be established by cytogenetic or molecular genetic studies. However, these highly specialized techniques take some days to be performed and are usually restricted to specialized haematology laboratories. Thus from a practical point of view morphology, cytochemistry and immunophenotyping are still important tools for rapid recognition of APL.

The great majority of APL cases with the $\mathrm{t}(15 ; 17)$ translocation are of the classic hypergranular M3 type and the microgranular (hypogranular) variant M3v, as described in the FrenchAmerican-British (FAB) classification $[5,6]$. Other morphological subtypes of APL have been reported: for example, hyperbasophilic [7], M1- and M2-like [8] and regular nuclear outline M3r [1]. While in the majority of classic APL cases the immunophenotypic pattern is distinctive, the features of the variant cases are more heterogeneous and nonspecific [9]. This case series is the first report from Qatar addressing the clinical-pathological profile of patients with APL.

\section{Methods}

\section{Patients}

All adult patients admitted to Al-Amal Hospital, the haematology/oncology centre of Hamad Medical Corporate in Qatar, and diagnosed with APL over the period January 2006 to May 2008 were included. Diagnosis of AML was established by combined morphological examination, immunophenotyping and cytogenetic studies.

\section{Clinical evaluation}

The medical records of the patients were reviewed for clinical manifestations of AML and results of laboratory tests. Complete and differential blood counts, full baseline biochemical profile, serum electrolytes, urea and creatinine, liver profile and coagulation screening profiles were done on all patients at presentation.

\section{Morphologic evaluation}

In 10 patients both peripheral smear and bone marrow aspirates (stained with Wright stain) were available, while in 1 patient diagnosis was based on the peripheral blood flow cytometry immunophenotyping as the patient died before there was time to perform bonemarrow aspiration. Differential counts of 100 cells on the peripheral smear and 500 cells on the bone marrow smear were performed. Final morphological subtype was determined by consensus between 2 haematopathologists.

\section{Cytogenetic analysis}

Cytogenetic analysis was performed on 10 of the 11 patients. Karyotypes were classified according to the International System for Human Cytogenetic Nomenclature (ISCN) [10].

\section{Flow cytometry immunophenotypic methods}

Bone marrow samples in 10 patients and peripheral blood in 1 patient were studied by flow cytometry using a panel of 19 monoclonal antibodies directly conjugated with fluorochrome.
A 3-colour flow cytometry analysis was performed on bone marrow aspirate/peripheral blood samples collected in EDTA tubes. After incubation of cells with monoclonal antibodies for 15 minutes at room temperature, the red blood cells were lysed with cyclic amine reagent (VersaLyse) for $10 \mathrm{~min}$ utes, followed by washing steps using phosphate-buffered saline solution. The cells were resuspended in phosphatebuffered saline.

The panel of antibodies included the following mouse monoclonal antibodies specific for: CD45 (energy-coupled dye $[E C D]$ ), CD34 (fluorescein isothiocyanate conjugated [FITC]), CD33 (phycoerythrin [PE]), CD64 (R-phycoerythrin covalently link to cyanin 5 [PC5]),CD13 (PE), CD14 (FITC), CD19 (FITC or ECD), CD117 (PE), CD10 (PE), CD7 (PE), CD3 (ECD), CD15 (FITC), HLA-DR (FITC), CD5 (FITC), TdT (FITC), cCD22 (PE), cMPO (FITC), cCD79a (PE) and CD20 (FITC). All antibodies were obtained from Beckman Coulter. For each antibody, negative staining levels were set by comparison with an isotype-matched control.

All samples were analysed with a FC500 flow cytometer (Beckman Coulter) equipped with an argon-ion laser with a wavelength of $488 \mathrm{~nm}$, by collecting at least 10000 ungated listmode events per tube. The cytometer was set up using standard operation procedures, and quality control was performed using the manufacturer's methods for calibration and compensation. Gating was based on the CD45 expression and side scatter. CXP software (Beckman Coulter) was used for data acquisition and analysis.

Cells were classified as positive for a given marker when the expression (fluorescence intensity) was greater than that of a negative (isotypic) control. Intensity of fluorescence was semi-quantitatively estimated as dim, moderate and high, based on the population position on the fluorescence scale. Antigen expression 
was considered to be homogenous if the distribution of the cells occupied up to 1 logarithmic decade on the scale of the fluorescence intensity, otherwise it was considered heterogeneous [11].

\section{Results}

Of the 34 patients diagnosed as AML between January 2006 and May 2008, 11 (32\%) were diagnosed with APL and were included in the study. Of these, 7 were men (1 Qatari and 6 non-Qatari) and 4 were women (1 Qatari and 3 non-Qatari). Their ages ranged from 16 years to 46 years,with a median age at presentation of 34.5 years.

\section{Clinical and laboratory features}

Of these 11 patients, 10 (91\%) presented with disseminated intravascular coagulation (DIC) according to the ISCN criteria [12] (Table 1). Gum bleeding, ecchymosis and symptomatic anaemia were presenting symptoms in 7 (63\%) patients, and haemoptysis, pulmonaryhaemorrhage and infection were found on diagnosis in $2(18 \%)$ patients; only 1 patient (9\%) had epistaxis on presentation and 1 presented with abdominal pain. None of the patients had organomegaly.

Severe thrombocytopenia $(<30 \times$ $10^{9} / \mathrm{L}$ ) was seen in 8 patients ( $\left.73 \%\right)$. Leukocytosis $\left(>10 \times 10^{9} / \mathrm{L}\right)$ was noted in 6 patients (55\%) and 5 (45\%) were severely anaemic at presentation. Pancytopenia was noted in 4 patients (36\%). In all, leukaemia promyelocytes were present in the peripheral blood (Table 1).

Only 2 patients (18\%) were classified as M3c, classic hypergranular APL, while 9 patients (82\%) had APL variants (Table 2).

The classic APL patients (patient nos. 1 and 2) presented with pancytopenia. The majority of the promyelocytes in the marrow were hypergranular; however, some hypogranular and agranular promyelocytes were also noted. Nuclear folding or convolution were frequently noted. Occasional faggot cells were seen in 1 patient (patient no. 1) (Figure 1A).

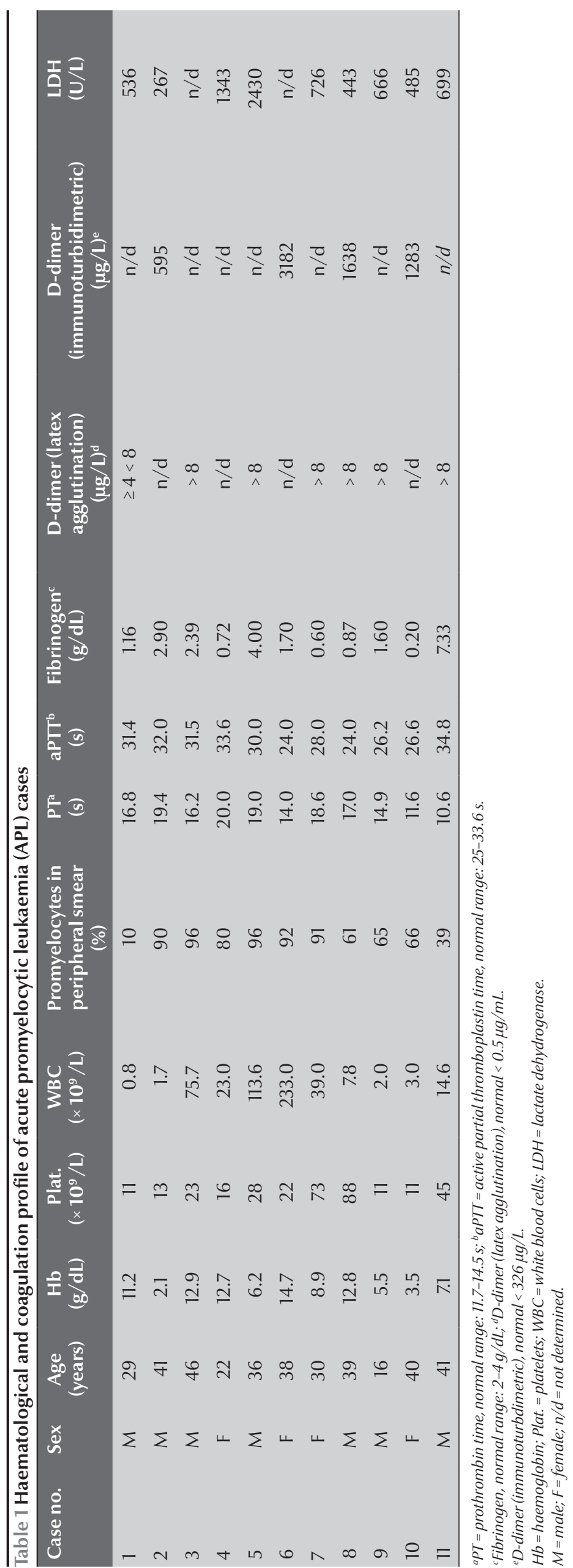


A

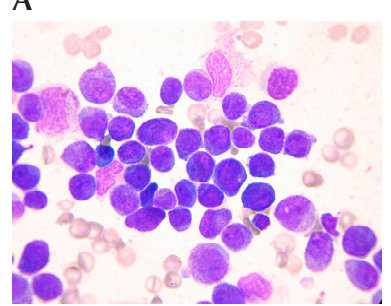

B

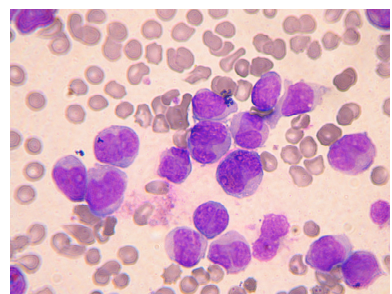

C

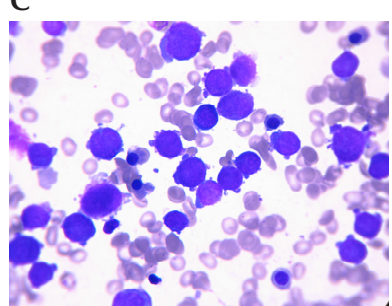

D

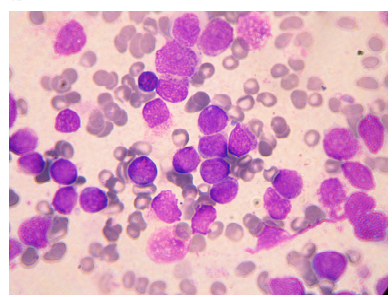

Figure 1 Bone-marrow smears of acute promyelocytic leukaemia (APL) cases. (A) Classic hypergranular APL in which hypergranular cells predominate and some cells show irregular nuclear outline. (B) Microgranular APL variant, where almost all the cells look agranular with prominent nuclear convolution. (C) Hyperbasophilic APL, where the majority of cells show hyperbasophilic cytoplasm with cytoplasmic projections. (D) M3r APL where most of the cells have round nuclei, both hypoand hypergranular cells are seen

In the 9 APL variant patients, 3 morphological categories were recognized. Microgranular variants M3v, as described by the French-AmericanBritish Co-operative Group [6] were recognized in 6 patients, with more than $50 \%$ of cells having agranular or hypogranular cytoplasm, with prominent nuclear folding. Bilobed cells were noted in 4 (patient nos. 3, 4, 6 and 8). Faggot cells were seen in 6 patients and were frequent in 1 of them (patient no. 3) (Figure 1B). In patients no. 9 and 10 the predominant cells were small in size with high nuclear-cytoplasmic ratio, deeply basophilic cytoplasm and showing prominent cytoplasmic blebs and projections, mostly agranular. Nuclear folding was also prominent with some bilobed cells and faggot cells noted in patient no. 9. These 2 cases correspond to the hyperbasophilic variant M3b of McKenna et al. [7] (Figure 1C). In the last patient (patient no. 11), a majority of the marrow promyelocytes (86\%) had regular, round or oval nuclei, hypergranularity was frequent (40\%) and faggot cells were occasionally noted, and APL was morphologically similar to the M3r regular variant reported by Sainty et al. [1] (Figure 1D).

\section{Cytogenetic analysis}

Out of the 10 patients analysed, 5 were positive for the $t(15 ; 17)$ translocation, 1 classic case (patient no. 2), 2 with M3-variant (patients no. 4 and 5) and 2 with hyperbasophilic APL. Additional cytogenetic abnormalities were noted in 2 cases (patients no. 2 and 5) (Table 2). In 3 cases the translocation was not detected and reported as normal karyotype. The marrow failed to culture in 2 patients.

\begin{tabular}{|c|c|c|c|c|c|c|c|c|}
\hline \multirow[t]{3}{*}{ Case no. } & \multirow{3}{*}{$\begin{array}{c}\text { Promy- } \\
\text { elocyte } \\
\%\end{array}$} & \multicolumn{2}{|c|}{ Cytoplasm } & \multicolumn{3}{|c|}{ Nucleus } & \multirow[t]{3}{*}{ Cytogenetic } & \multirow{3}{*}{$\begin{array}{c}\text { APL } \\
\text { subtype }\end{array}$} \\
\hline & & Hypergranular & $\begin{array}{c}\text { Hypo/ } \\
\text { agranular }\end{array}$ & Regular & Folded & Bilobed & & \\
\hline & & $\%$ & $\%$ & $\%$ & $\%$ & $\%$ & & \\
\hline 1 & 90 & 65 & 35 & 14 & 69 & 17 & Failed & M3c \\
\hline 2 & 85 & 68 & 32 & 59 & 34 & 7 & $\begin{array}{l}46, X Y, \operatorname{der}(4), t(4 ; 7) \\
(q 31 ; q 22), t(15 ; 17) \\
(q 22 ; q 21), \operatorname{del}(16)(q 22)[12]\end{array}$ & $\mathrm{M} 3 \mathrm{c}$ \\
\hline 3 & 96 & 3 & 97 & 12 & 57 & 31 & $46, X Y[16]$ & $M 3 v$ \\
\hline 4 & 90 & 14 & 86 & 20 & 59 & 21 & $46, X X, t(15,17)(q 22 ; q 12)[15]$ & $M 3 v$ \\
\hline 5 & 92 & 38 & 62 & 22 & 68 & 10 & $\begin{array}{l}46, X Y, \operatorname{del}(3)(q 12 q 23) \\
t(15 ; 17)(q 22 ; q 12)[26]\end{array}$ & M3v \\
\hline $6^{\mathrm{a}}$ & $\mathrm{n} / \mathrm{d}$ & 0 & 100 & 10 & 46 & 44 & $\mathrm{n} / \mathrm{d}$ & $M 3 v$ \\
\hline 7 & 92 & 21 & 79 & 29 & 66 & 5 & $46, X X[20]$ & M3v \\
\hline 8 & 83 & 29 & 71 & 7 & 70 & 23 & Failed & M3v \\
\hline 9 & 78 & 11 & 89 & 15 & 77 & 8 & $46, \mathrm{XY}, \mathrm{t}(15 ; 17)[14]$ & $\mathrm{M} 3 \mathrm{~b}$ \\
\hline 10 & 75 & 20 & 80 & 5 & 90 & 5 & $46, X X, t(15 ; 17)(q 22 ; q 11.2)[11]$ & M3b \\
\hline 11 & 82 & 35 & 65 & 86 & 12 & 2 & $46, X Y[27]$ & $\mathrm{M} 3 \mathrm{r}$ \\
\hline
\end{tabular}

For descriptive purposes, morphologic variations were grouped into different subtypes: $M 3 \mathrm{C}=$ hypergranular classic; $M 3 \mathrm{v}=$ microgranular variant; $\mathrm{M} 3 \mathrm{~b}=$ hyperbasophilic variant; M3r= microgranular regular. Within the M3v group cases are arranged chronologically. $n / d=$ not determined .

${ }^{a}$ Done on peripheral blood. 

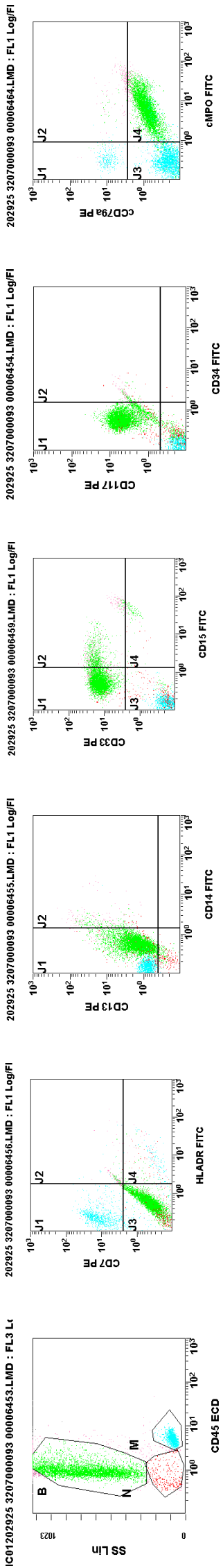
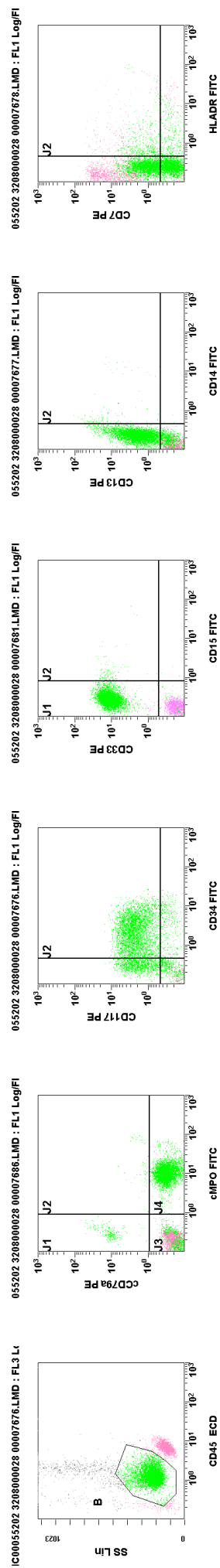

\section{Immunophenotyping}

The expression pattern of the selected markers studied by flow cytometry analysis and their correlation with the morphological subtypes are summarized in Table 3.

On side-scatter versus CD45 leukaemic cells in the classic hypergranular patient displayed primarily high sidescatter (Figure 2A). In contrast, 8 of the 9 M3-variant patients (89\%), the cells displayed less side-scatter and generally fell in the area closer to the blast region (Figure 2B). Myeloperoxidase (MPO) protein and CD33 were consistently expressed in all the cases (100\%) regardless of the subtype. CD33 expression was homogenous in 10 patients (91\%) and heterogeneous in 1 patient (patient no. 5). CD 13 was positive in 10 patients (91\%) and CD 117 in 9 patients (82\%). CD13 expression was heterogeneous in all the positive patients, CD34 was positive in 4 patients (36\%); all were of the variant type (patients no. 3, 6, 9 and 10). Each of CD14 and CD15 was positive in 2 patients (18\%). CD64 was positive in 6 out of the 8 cases where it was done (75\%); 1 of them (patient no. 2) coexpressed CD 14 as well. The human leukocyte antigen DR (HLADR) molecule was expressed in 1 case (patient no. 9) and CD7 in 1 case (patient no. 10).

TdT, CD3, CD19, CD10, CD5, CD20, CD79 and CD22 were negative in all the cases.

\section{Discussion}

The methods used for the diagnosis and classification of acute leukaemias include morphology, cytochemistry, immunophenotyping, cytogenetic and molecular genetics [13]. For treatment purposes, once the separation between myeloid and lymphoid leukaemias has been solved, the most important definition is if a given case of acute myeloid leukaemia could be sub-classified as 


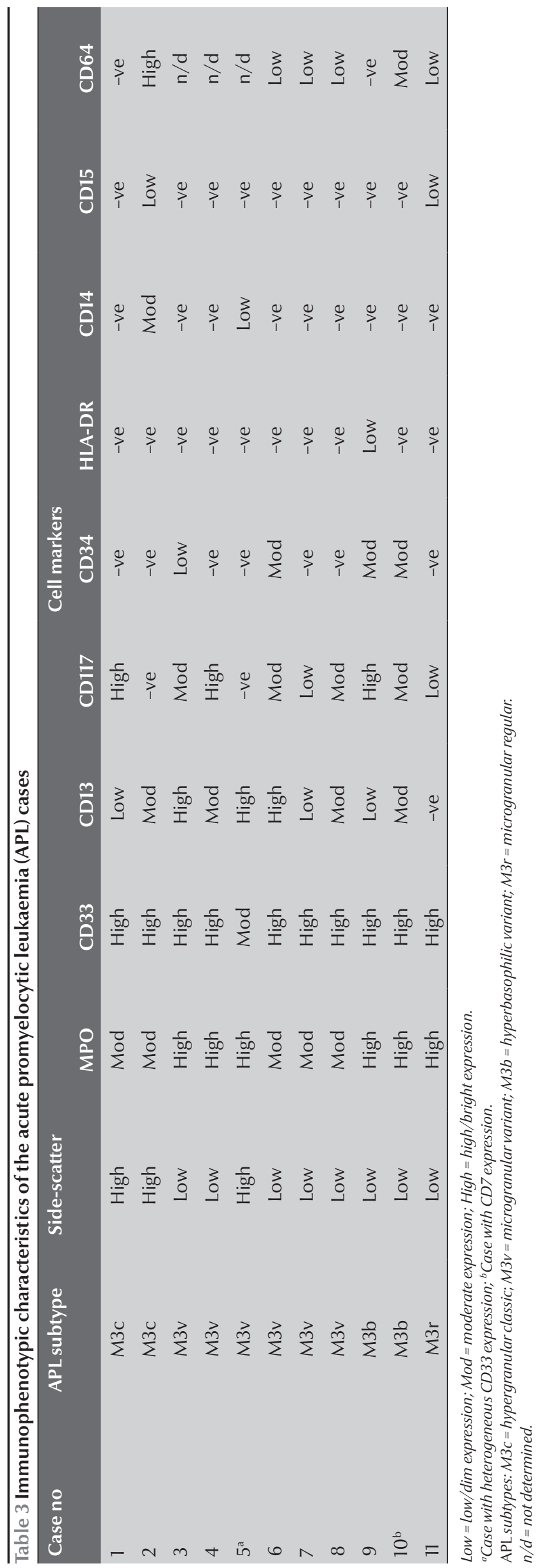

APL, since this subtype benefits from treatment with ATRA.

Although the use of morphology for initial diagnosis sounds appealing, there are some problems when only this method is used. In the recovery phase from acute agranulocytosis, for example, the bone marrow may be replenished with promyelocytes, displaying a picture similar to classic APL [14]. Microgranular APL may also be confused with other subtypes of AML, mainly acute monocytic leukaemias [15]. Furthermore some groups have described other morphological subtypes of APL in addition to the classic and microgranular forms - such as the hyperbasophilic, the M1-like and M2-likethat display a morphological picture quite different from classic APL and are difficult to distinguish from M7, M1 and M2 AML respectively $[7,8]$.

Although our series included a small number of cases, it seems that APL is a common subtype (32\%) among AML cases in Qatar, higher than the frequency of $5 \%-10 \%$ reported from other countries $[16,17]$ and the frequency of $17 \%$ from Saudi Arabia in a series including both Saudi and nonSaudi patients [18] and 10\% in Omani patients [19]. A higher frequency of APL has been noted in certain ethnic groups such as Latin American and Spanish populations, which have a significantly higher frequency of the $P M L / R A R a$ gene [caused by fusion of the promyelocytic leukaemia (PML) gene and the gene encoding the retinoic acid receptor- $\alpha(R A R \alpha)$, which may suggest a possible influence of genetic and/or environmental factors [20]. In our series $82 \%$ of patients were nonQatari, and were of several different nationalities, which makes the possibility of a link to a genetic factor rather unlikely and this high frequency needs to be verified on a larger series over a longer period.

The other interesting finding was the predominance of the variant APL which we found in $82 \%$ of our patients in contrast to the wellknown notion that variant APL cases account for only about $15 \%-20 \%$ of all APL in adults $[1,2]$. This would explain the high frequency of high white blood cell count (55\%), severe thrombocytopenia (73\%) and DIC (91\%), features reported to be more commonly 
encountered in variant APL cases and all of which have been shown to be unfavourable prognostic factors [21].

Immunophenotypic studies have unveiled a characteristic pattern in classic APL compared with other subtypes of AML, which includes expression of MPO, CD33, CD13 and lack of HLADR and CD34 [9]. This distinctive pattern was also displayed by the promyelocytes in our patients with classic APL morphology. On the other hand the pattern in variant APL has been reported to be more heterogeneous, with a higher percentage of cells expressing the T-cell antigen CD2, stem cell marker CD34 [9], HLA-DR [21] and CD56 [1]. This heterogeneity was demonstrated in our variant APL patients as 4 expressed CD34 (2 M3v and 2 hyperbasophilic subtypes). CD34 expression was suggested as a reliable marker to distinguish between M3v and classic APL [9]. HLA-DR was expressed in 1 patient with hyperbasophilic APL. Fenu et al. reported 2 cases of hyperbasophilic APL variant with positive expression of CD33, CD13, HLA-DR, CD34, CD2 and CD9 [22].

CD 14 was expressed in $1 \mathrm{M} 3 \mathrm{v}$ patient and CD64 in 5 out of the 6 variant patients tested (3 M3v, 1 hyperbasophilic and $1 \mathrm{M} 3 \mathrm{r}$ types). Both of these are monocytic markers which add to the difficulty in recognizing $\mathrm{M} 3 \mathrm{v}$ APL as it might simulate monocytic leukaemia, not only morphologically but immunophenotypically as well.

The $\mathrm{t}(15 ; 17)$ translocation yielding the PML-RARa fusion gene is the diagnostic hallmark of APL. This translocation can be detected by conventional cytogenetic techniques in about $90 \%$ of cases. In the majority of cases lacking the $t(15 ; 17)$ translocation, PML-RARa gene rearrangement can be detected by molecular analysis created by insertion or more complex rearrangements. Such mechanisms occur in approximately $4 \%$ and $2 \%$ of APL cases respectively. In a minority of APL cases other rearrangements of genes were reported, whereby the $R A R a$ gene is fused to the promyelocytic leukaemia zinc finger (PLZF) gene in about $0.8 \%$ of cases and less commonly to nucleophosmin (NPM), nuclear matrix associated (NuMA) and signal transducer and activator of transcription $5 \mathrm{~b}$ (STAT56) genes [23]. Many of the cases with the latter molecular defects show clinical and cytological differences from the M3/M3v subtypes of AML, and the designation "M3-like" has been suggested [24]. In common with PML-RARa-associated APL, patients with fusion genes involving NPM and NuMA appear to be sensitive to ATRA. In contrast, APL with PLZF/RARa or $S T A T 5 b / R A R a$ rearrangements are typified by a lack of response to retinoids.

In this series classic $\mathrm{t}(15 ; 17)$ was demonstrated in 5 out of the 8 APL variant cases analysed (63\%). Analysis of 3 cases revealed a normal karyotype. Whether the lower than expected rate of $t(15 ; 17)$ expression is another unique feature of APL in Qatar, like the morphological diversity, or whether it is a chance finding needs to be verified on a larger number of cases.

In conclusion, APL seems to constitute a major proportion of AML cases in Qatar, with significant morphological heterogeneity and predominance of variant APL cases with unfavourable presenting features. The impact of these findings on patient outcomes is being evaluated and will be reported.

The heterogeneous pattern and limitations of flow cytometry in diagnosing APL variants, as confirmed in this study, emphasize the importance of a careful morphological evaluation with good awareness of the cytological spectrum of APL, especially in this country where $t(15 ; 17)$ is not frequently detected.

The study also highlighted the importance of more sensitive approaches to APL diagnosis, such as interphase FISH [fluorescent in situ hybridization] reverse transcriptase (RT)-PCR molecular analysis or the interesting option for a rapid and accurate diagnosis of APL by immunostaining assays with anti-PML antibodies to detect the characteristic microparticulate nuclear pattern of the PML protein. The latter may be an important tool for identifying cytologically atypical APL cases and in monitoring patients for minimal residual disease, particularly in our institution where genetic tests for acute leukaemias are not yet routinely available.

\section{Acknowledgements}

We would like to express our deep thanks to following colleagues for help in performing this study: Halima El-Omri, Ruba T. Yassin, Reeham N. Hasssan, Ussama Al-Homsi, Zainab M. Fawzi, Munir Jalis, Kamal R. Abboudi.

\section{References}

1. Sainty D et al. A new morphologic classification system for acute promyelocytic leukemia distinguishes cases with underlying PLZF-RARa gene rearrangements. Blood, 2000, 96:1287-1296.

2. Avvisati G, Lo Coco F, Mandelli F. Acute promyelocytic leukemia: clinical and morphologic features and prognostic factors. Seminars in Hematology, 2001, 38:4-12.
3. Fenaux $\mathrm{P}$, Chromienne $\mathrm{C}$, Degos L. All-trans retinoic acid and chemotherapy in the treatment of acute promyelocytic leukemia. Seminars in Hematology, 2001, 38:13-25.

4. Barbui T, Finazzi G, Falanga A. The impact of all-trans-retinoic on the coagulopathy of acute promyelocytic leukemia. Blood, 1998, 91:3093-3102. 
5. Bennett JM et al. Proposals for the classification of the acute leukemias. FAB Cooperative Group. British Journal of Haema tology, 1976, 33:451-458.

6. Bennett JM et al. A variant form of hypergranular promyelocytic leukaemia M3. British Journal of Haematology, 1980, 44:169-170.

7. McKenna RW et al. Acute promyelocytic leukaemia: a study of 39 cases with identification of a hyperbasophilic microgranular variant. British Journal of Haematology, 1982, 50:201-214.

8. Neam PB et al. Morphology of acute promyelocytic leukaemia with cytogenetic or molecular evidence for the diagnosis: characterization of additional microgranular variants. American Journal of Hematology, 1997, 56:131-142.

9. Paietta E et al. A surrogate marker profile for PML/RAR. Expressing acute promyelocytic leukemia and the association of immunophenotypic markers with morphologic and molecular subtypes. Cytometry Part B Clinical Cytometry. 2004, 59B:1-9.

10. Mittleman F ed. ISCN 1995: An international system for human cytogenetic nomenclature. Basel, Karger, 1995.

11. Gorczyca W, ed. Flow cytometry in neoplastic hematology: morphologic-immunophenotypic correlation. London, Taylor and Francis, 2006:1-20.

12. Taylor FB Jr et al. Towards definition, clinical and laboratory criteria, and a scoring system for disseminated intravascular coagulation. Thrombosis and Haemostasis, 2001, 86:1327-1330.

13. McKenna RW. Multifaceted approach to the diagnosis and classification of acute leukaemias. Clinical Chemistry, 2000, 46:1252-1259.

14. Rizzatti EG et al. Expression of CD117 and CD11b in bone marrow can differentiate acute promyelocytic leukaemia from recovering benign myeloid proliferation. American Journal of Clinical Pathology, 2002, 118:31-37.

15. Nagendra $S$ et al. Leukemias resembling acute promyelocytic leukemia, microgranular variant. American Journal of Clinical Pathology, 2002, 117:651-657.
16. Ghosh $\mathrm{S}$ et al. Haematologic and immunophenotypic profile of acute myeloid leukemia: an experience of Tata Memorial Hospital. Indian Journal of Cancer, 2003, 40 2:71-76.

17. Arber DA et al. Prognostic impact of acute myeloid leukemia classification. Importance of detection of recurring cytogenetic abnormalities and multilineage dysplasia on survival. American Journal of Clinical Pathology, 2003, 119:672-680.

18. Harakati MSE et al. Adult acute myeloblastic leukemia: experience at King Khalid University Hospital. Annals of Saudi Medicine, 1998, 18:221-225.

19. Udayakumar AM et al. Cytogenetic, morphologic and immunophenotypic patterns in Omani patients with de novo acute myeloid leukemia. Cancer Genetics and Cytogenetics, 2007, 177:89-94.

20. Ribeiro RC, Rego E. Management of APL in developing countries: epidemiology, challenges and opportunities for international collaboration. Hematology, 2006, 1:162-168.

21. Olga F et al. APL: a classic tale of bench to beside. In: Karp J. Acute myelogenous leukemia. Totowa, New Jersey, Humana Press, 2007:193-231.

22. Fenu S et al. P21. Acute promyelocytic leukemia: two cases with cryptic aspects in morphological diagnosis. Haematologica Reports, 2005, 1(7):46.

23. Grimwade D et al. Characterization of acute promyelocytic leukemia cases lacking the classic $\mathrm{t}(15 ; 17)$ : results of the European Working Party. Blood, 2000, 96(4):1297-1308.

24. Acute leukaemia: immunophenotypic, cytogenetic and molecular genetic analysis in the classification of acute leukaemia-the EGIL, MIC, MIC-M and WHO classifications. In: Bain BJ, ed. Leukaemia diagnosis, 3rd ed. Oxford, Blackwell Publishing, 2003:57-144. 